Bundesgesundheitsbl 2014 · 57:1243-1245

DOI 10.1007/s00103-014-2070-9

Online publiziert: 17. Oktober 2014

(c) Springer-Verlag Berlin Heidelberg 2014

Osamah Hamouda ${ }^{1} \cdot$ Gérard Krause $^{2} \cdot$ Iris Pigeot $^{3}$

${ }^{1}$ Robert Koch-Institut, Abt. für Infektionsepidemiologie, Berlin, Deutschland

${ }^{2}$ Abteilung Epidemiologie, Helmholtz Zentrum für Infektionsforschung (HZI), Braunschweig, Deutschland

${ }^{3}$ Leibniz-Institut für Präventionsforschung und Epidemiologie - BIPS, Bremen, Deutschland

\title{
Nationale Kohorte - die größte Gesundheitsstudie Deutschlands
}

Noch in der zweiten Hälfte des 19.Jahrhunderts gehörten in allen Teilen der Welt Tuberkulose, Pocken, Durchfallerkrankungen, Typhus und Diphterie zu den Haupttodesursachen. Heute haben Infektionskrankheiten in Industrieländern als unmittelbare Ursachen für Morbidität und Mortalität eine geringere Bedeutung. Zugleich jedoch besteht Grund zur Annahme, dass Infektionskrankheiten als prädisponierende Faktoren für die Entstehung nichtinfektiöser chronischer Krankheiten eine deutlich unterschätzte und nur schwer zu erforschende Rolle spielen. In der Nationale Kohorte ist die Erfassung von Infektionen und ihren Risikofaktoren sowie ihrer Rolle bei der Entstehung chronischer Krankheiten von besonderem Interesse.

Das vorliegende Heft stellt in zahlreichen Beiträgen die Ergebnisse der ersten Machbarkeitsstudien (Pretests) für den Bereich Infektionskrankheiten im Rahmen der Nationalen Kohorte dar. Ein Ziel von Pretests ist es, Arbeitsabläufe zu entwickeln, sie zu testen und auf ihre Machbarkeit hin zu prüfen. Dies betrifft insbesondere das Management von Bioproben und Daten sowie die Qualitätskontrolle. Des Weiteren werden verschiedene Instrumente zur Datenerhebung und medizinische Untersuchungsgeräte auf ihre Verwendbarkeit und Praktikabilität in großen populationsbezogenen Kohortenstudien getestet. Auch die Akzeptanz der Probanden wird im Vorfeld untersucht, um die Auswahl der am besten geeigneten Instrumente zu ermöglichen und vorhandene Instrumente speziell für die Studienzwecke anzupassen. Hintergrundinformationen zu den Pretests der Nationalen Kohorte findet man in dem Beitrag von W.
Ahrens und Koautoren zum Design der Machbarkeitsstudien.

Um Infektionsforschung gemeinsam an vielen verschiedenen Standorten durchführen zu können und um valide Ergebnisse aus dieser Forschung zu gewinnen, liefert eine umfassende gut funktionierende Biobank-Struktur einen wichtigen Beitrag. Biomaterialien mit groBer Relevanz für den Bereich Infektionskrankheiten sind zum Beispiel Blut, Urin, Speichel, cervicovaginale Flüssigkeit und Stuhlproben sowie Nasen- und Rachenabstriche. Um die Machbarkeit von Probensammlungen und -präparation sowie den Transport und die Lagerung der Proben zu überprüfen, wurden in zwei aufeinanderfolgenden Pretests 1.600 aus der Bevölkerung ausgewählte Teilnehmer gebeten, Probenmaterial zu spenden. Die durchschnittliche Teilnahmequote an der Sammlung von Biomaterialien, die A. Kühn und Koautoren beschreiben, betrug $95 \%$. Es zeigte sich zudem, dass die entwickelten Standard-Arbeitsanweisungen umsetzbar und mit kleinen Anpassungen und Modifikationen für die Hauptphase der Studie einsetzbar sind.

Zusätzlich zur Biobank-Struktur ist auch die Akzeptanz der Probengewinnung durch die Teilnehmer ein wichtiger Faktor für eine erfolgreiche Studiendurchführung. Insbesondere bei der Gewinnung von Stuhlproben und cervicovaginaler Flüssigkeit sind Voruntersuchungen erforderlich, um hier die höchstmögliche Akzeptanz innerhalb der Teilnehmergruppe zu erreichen. Zur Durchführung bestimmter Laboranalysen wird $\mathrm{Na}$ tivstuhl benötigt, der zeitnah vor Ort oder in einem nahegelegenen Labor verarbeitet werden muss. Bislang ist nicht bekannt, ob es möglich ist, Stuhlproben von gesunden 
Probanden während ihres Aufenthaltes im Studienzentrum zu erhalten. A. Schultze und Koautoren haben daher in ihrem Pretest die Machbarkeit und die Akzeptanz der Stuhlprobengewinnung während eines Termins im Studienzentrum mit der Stuhlprobengewinnung zu Hause verglichen. Vor die Wahl gestellt, haben sich die meisten Probanden in diesem bevölkerungsbasierten Pretest für die Gewinnung von Stuhlproben zu Hause statt im Studienzentrum entschieden. Die Heimgewinnung hat sich als sehr zuverlässige Methode für Fragestellungen erwiesen, die keine frisch gewonnenen Nativstuhlproben erfordern. Für die Nativstuhlproben zur sofortigen Verarbeitung im Studienzentrum konnte nur eine kleine Gruppe von Probanden gewonnen werden, was möglicherweise zu Selektionsverzerrungen führen könnte. Es sollen weitere Studien folgen, um die möglichen Selektionseffekte näher zu untersuchen.

Während die Frauengesundheit im Basisinterview thematisiert wird, ist der direkte Erregernachweis bei cervicovaginalen mikrobiellen Besiedlungen oder Infektionen nicht Teil des Untersuchungsprotokolls. In einem Pilotprojekt hat deshalb das Team um S. Castell die Machbarkeit der häuslichen Selbstentnahme einer cervicovaginalen Lavage durch Studienteilnehmerinnen der Nationalen Kohorte ohne Kontakt zu einem Frauenarzt oder anderem medizinischen Personal untersucht. Das Team hat außerdem die Fähigkeit des Vorgehens exploriert, verschiedene vaginal auftretende Erreger und Erkrankungen, inklusive humane Papillomviren (HPV), Chlamydia trachomatis und bakterielle Vaginose (BV) nachzuweisen. Die Analyse der Akzeptanzfragebögen im Pretest ergab, dass die selbstständige Durchführung einer cervicovaginalen Lavage für $98 \%$ der Frauen akzeptabel war, dass $89 \%$ die Probenentnahme als leicht und $96 \%$ als benutzerfreundlich einstuften. Die Machbarkeitsstudie zeigte außerdem, dass aus den gewonnenen cervicovaginalen Flüssigkeiten erfolgreich Pathogennachweise durchgeführt werden konnten. Sollte die cervicovaginale Probenselbstentnahme zukünftig Anwendung in der Nationalen Kohorte finden, würde dies die Untersuchung der Transienz/Persistenz oder langfristiger Effekte vaginaler (Ko-) Infektionen und Kolonisationen ermöglichen.

Katzen und Hunde leben in mehr als $20 \%$ der deutschen Haushalte und der Kontakt zwischen diesen Kleintieren und ihren Haltern kann sehr eng sein, sodass es hier zu einer wechselseitigen Übertragung von Erregern kommen kann. In den letzten Jahren traten verstärkt Hinweise auf, dass Haustiere Überträger von Methicillin-resistenten Staphylococcus-aureus-Bakterien sein können, die gegen die meisten heute verfügbaren Antibiotika unempfindlich sind. Daher ist es wichtig zu erfahren, wie die Transmissionsdynamik von zoonotischen Erregern ist, welche Faktoren die Übertragung der Erreger zwischen Haustier und Halter beeinflussen sowie ob solche Übertragungen bei der Entwicklung von chronischen Krankheiten eine Rolle spielen. Um festzustellen, ob diese Zoonose-Forschungsfragen in bevölkerungsbasierten Studien wie der Nationalen Kohorte untersucht werden können, wurden zwei Studien an unterschiedlichen Studienpopulationen im Rahmen der Machbarkeitsstudien der Nationalen Kohorte durchgeführt. Die erste Studie hat gezeigt, dass die Exposition der Teilnehmer der Nationalen Kohorte gegenüber Hunden und Katzen bei 10 bzw. $14 \%$ liegt. In der zweiten Studie, die hier von K. Hille und Kollegen zusammenfassend dargestellt wird, wurde die Machbarkeit der Beprobung von Hunden und Katzen durch ihre Besitzer getestet und ob deren Qualität vergleichbar mit der von Proben ist, die von einem qualifizierten Tierarzt genommen wurden. Den Tierbesitzern wurden Anleitungen zur Probenentnahme und zum Probenentnahmematerial für Nasenabstriche, Maulschleimhautabstriche und Kotproben mitgegeben. Die Abstriche wurden in der Kultur untersucht und das Bakterienwachstum wurde unabhängig von der Bakterienspezies quantifiziert. Es wurde das Wachstum grampositiver und gramnegativer Bakterien von Proben, die durch den Tierarzt bzw. durch den Tierbesitzer genommen wurden, miteinander verglichen. Der Pretest zeigte, dass die Beprobung durch die Tierhalter ein geeignetes Tool ist, um in der Nationalen Kohorte Anwendung zu finden und so zur Klä- rung von Zoonose-Forschungsfragen beizutragen.

In der Nationalen Kohorte werden zusätzlich zur Bioprobengewinnung Fragebögen verwendet, um Daten zu ausgewählten Infektionskrankheiten und ihren Symptomen sowie zu Daten zum Impfstatus der Teilnehmer zu erfassen.

C. Sievers und Kollegen haben im Pretest einen Fragebogen auf Machbarkeit und Vollständigkeit evaluiert, der Infektionen und medizinische Behandlungen mittels Selbstangabe und Selbsteinschätzung der Teilnehmer erfasst. Insgesamt wurde der Fragebogen von den Teilnehmern als gut verständlich eingestuft. Jedoch zeigte sich, dass medizinische Begriffe und seltene Erkrankungen noch verständlicher erklärt werden müssen.

Das Team um A. Schultze überprüfte verschiedene Aspekte der Impfdatenerhebung auf Akzeptanz und Machbarkeit im Rahmen des Pretests der Nationalen Kohorte. Zum einen wurde getestet, ob eine grundsätzliche Bereitschaft der Probanden besteht, die Impfdaten unter Zuhilfenahme der Impfpässe in den Studienzentren zu erheben. Es zeigte sich, dass eine hohe Bereitschaft und Zuverlässigkeit der Probanden besteht, die Impfpässe im Studienzentrum vorzulegen und dass auf diese Weise eine verlässliche Impfdokumentation im Rahmen der Nationalen Kohorte erfolgen kann. Zum anderen wurde überprüft, ob eine zusätzliche Erinnerung die Bereitstellung der Impfpässe positiv beeinflusst. Das Versenden einer Erinnerungskarte hat sich zwar, unabhängig davon, ob sie zusammen mit der Terminbestätigung oder separat übermittelt wurde, positiv, aber nicht bedeutend auf das Mitbringen der Impfausweise ausgewirkt. Daher empfiehlt der Beitrag von A. Schultze und Koautoren die standardmäßige Entsendung von separaten Erinnerungspostkarten nicht. Vielmehr halten sie die Ergänzung eines Satzes zum Thema „Mitbringen der Impfpässe “ in der Terminbestätigung für ausreichend. Des Weiteren wurde die Möglichkeit der zusätzlichen Validierung über die hausärztliche Dokumentation geprüft. Der Datenerhebung über den Hausarzt stimmten $62 \%$ aller Pretest-Teilnehmer zu. Von den angeschriebenen Hausärzten schickten jedoch nur $43 \%$ der Ärzte die angeforder- 
ten Impfdaten zurück. Nur für ungefähr ein Viertel aller Probanden erscheint die zusätzliche Validierung über die hausärztliche Dokumentation somit möglich. Die Autoren empfehlen aufgrund der knappen zeitlichen und personellen Ressourcen, nur Personen um Einwilligung zur Kontaktaufnahme mit dem Hausarzt zu bitten, die keinen Impfausweis besitzen, oder bei spezifischen Forschungsfragen, wie zum Beispiel zur Influenza-Impfung, die nicht im Impfausweis vermerkt wird.

S. Castell und Kollegen evaluierten im Pretest die neu ergänzten Fragebogenitems zur selbst eingeschätzten Anfälligkeit gegenüber verschiedenen Infektionskrankheiten und prüften ebenfalls die Test-Retest-Reliabilität des Fragebogens. Grundsätzlich ist dieser Fragebogen zu selbst berichteten Infektionskrankheiten ein relativ zuverlässiges Instrument und kann daher in weiteren Studien zu Infektionskrankheiten angewendet werden. Das Instrument zur Selbsteinschätzung der Anfälligkeit gegenüber verschiedener Infektionskrankheiten muss hingegen weiter auf Eignung und Einsatzfähigkeit geprüft werden.

In der heutigen Zeit ist die Nutzung des Internets für viele Personen in Deutschland alltäglich. Dies zeigt sich auch daran, dass im Jahr 2013 insgesamt $82 \%$ aller Haushalte in Deutschland über einen Internetzugang verfügen. Daher beschäftigte sich eine Machbarkeitsstudie, die in dem Beitrag von S. Mall und Koautoren näher beschrieben wird, auch mit der Nutzung einer Web-basierten Erhebung. Im Rahmen dieser Studie wurde untersucht, ob Daten zu akuten respiratorischen und gastrointestinalen Erkrankungen durch einen Web-basierten Fragebogen über einen längeren Zeitraum erhoben werden können. Hierfür erhielten die Teilnehmer jede Woche eine E-Mail, die an die wöchentliche Teilnahme erinnert. Die wöchentlichen Rückmeldungsraten variierten zwischen 62 und $81 \%$, der Median betrug $74 \%$. Eine Erfassung mithilfe von Web-basierten Fragebögen erscheint daher sehr gut durchführbar. Die Anzahl der Studienteilnehmer mit Internetanschluss war hoch und es traten keine größeren technischen Probleme in der Durchführung auf. Es zeigte sich jedoch auch, dass die Teilnehmer an der Mach- barkeitsstudie jünger und besser gebildet waren als die Nichtteilnehmer. Daher muss die Möglichkeit einer Verzerrung berücksichtigt werden, wenn es um die Übertragbarkeit der Ergebnisse auf die Allgemeinbevölkerung geht.

Die Nationale Kohorte kann zur Gewinnung von neuen Erkenntnissen zum Zusammenwirken sozialer, psychischer, biomedizinischer, umweltbedingter und Lebensstilfaktoren bei der Entstehung, Behandlung und Prävention von Krankheiten einen entscheidenden Beitrag leisten. Insbesondere der Umstand, dass Daten zu sozialen Faktoren und zum Lebensstil in Verbindung mit biomedizinischen und versorgungsbezogenen Parametern gemeinsam erhoben und untersucht werden können, bietet zusammen mit der großen Teilnehmerzahl und einer langen Beobachtungszeit eine einmalige Möglichkeit komplexe Wirkungszusammenhänge aufzudecken. Damit trägt die Nationale Kohorte dazu bei, die Gesundheit jedes einzelnen zu erhalten und einer Erkrankung frühzeitig vorzubeugen.

Mit der Zuversicht, dass diese Forschungsinitiative die Epidemiologie in Deutschland weiter voranbringen und an die internationale Spitzenforschung anknüpfen wird, verbleiben wir.

Ihre

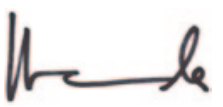

Osamah Hamouda

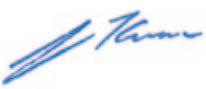

Gérard Krause

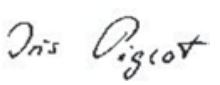

Iris Pigeot

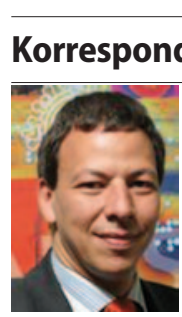

Robert Koch-Institut, Abt. für Infektionsepidemiologie Seestraße 10, 13353 Berlin HamoudaO@rki.de

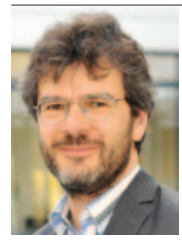

Prof. Dr. G. Krause

Abteilung Epidemiologie Helmholtz Zentrum für Infektionsforschung (HZI) Inhoffenstraße 7 38124 Braunschweig gerard.krause@helmholtzhzi.de

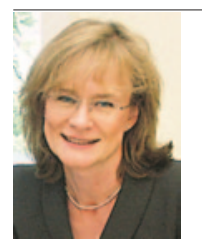

Prof. Dr. I. Pigeot Leibniz-Institut für Präventionsforschung und Epidemiologie - BIPS

Achterstraße 30

28359 Bremen

pigeot@bips.uni-bremen.de

\section{Einhaltung ethischer Richtlinien}

Interessenkonflikt. O. Hamouda, G. Krause und I Pigeot geben an, dass kein Interessenkonflikt besteht.

Dieser Beitrag beinhaltet keine Studien an Menschen oder Tieren. 